\title{
Few Reflections toward the Cancer Problem
}

\section{Daniel Gandia and Juan Manuel Carrera*}

IQVIA, Hematology and Oncology Strategy Unit Buenos Aires, Argentina

*Corresponding Author: Juan Manuel Carrera, IQVIA, Hematology and Oncology Strategy Unit Buenos Aires, Argentina.
Received: May 16, 2021

Published: July 21, 2021

(C) All rights are reserved by Daniel Gandia and Juan Manuel Carrera .
Cancer is an example of disease that fascinates Basic and Clinical Researchers. We don't know at the present time, if most malignant tumors are, a) "early genetic programmed diseases", b) the result of a random-related process of biological, chemical or physical impacts to genes during life, c) or a combination of both.

If a) is the case, the cell must comport peculiar parts of its genome programmed for the development of the whole Cancer process.

These genetic programs must be complex and maximally penetrant for a phenotypic trait as is the information for a sick cell migratory program: the metastases. Migration is common in higher tissue cells and a process during the embryonic period that helps in the posterior differentiation of the ectodermal, mesodermal and endodermal layers (all tissues origin). In normal early migration, cells know where to go and where to spatially stop, which says a lot about the nicely designed "normal" process; it can start at it's own time, knows where and where not to express particular gene patterns; and the ability to stop it, whenever is needed. Having this potential within a regular cell is key to understand what goes wrong outside this normal situation. Cancer cells do not $\mathrm{t}$ spatially stop, but know where to go outside its primary site. We can't consider this a milestone only related to cell itself, no cell is alone within a living tissue and of course in a living environment. All this constant and cumulative interaction is highly involved and mainly responsible for different and potential cell differentiation.

Considering b) and c), the committed cell reprogrammed its life, in order to survive and persist after many impacts and adaptations in another living organization level, with altered migratory genetic and cell division programs. Adapting is a key point.
"DNA malignization", as a concept, involves the need of using same kind of gene machinery, such activation of protooncogenes in oncogenes and loss of tumor suppressor genes for a cancer cell to survive. Machinery widely used in normal cell cycles, but required for regular embryofetal development: transcription factors, cell receptors or signaling molecules; all with the final impact of a new pattern of gene expression. Mutations may vary, and include gene amplifications, gene deletions, gene duplications such a somatic copy number alterations, this last one pervasive than the others and conducing to altered chromatin structural regulation, chromosomal instability, and karyotype order. Like this, malignant tumors present different chromosomes number alterations (mainly aneuploidy) and altered structures due to the "malignant genic" process.

This last, present the driver oncogenes for the cell limitless division, cell differentiation and absence of programmed death.

The clue issue are tumor suppressors genes. We know partially how they work. Cancer arises, once cell lost both functional copies. Oncogenic process depends on the loss of function of this gene. Therefore, there is no expression at all, which make it a more difficult target to be considered as druggable., unlike oncogenes. After oncogenic driver mutations are stablished and a cell is considered malignant, tumor suppression genes function becomes absent.

One of the great challenges in Cancer drugs discovery, is the development of "enhancers" of tumor suppressor genes. There is limited and not consistent data about targeting p53. Examples includes; but no stop after limiting and vaccines, targeting downstream mutated p53, gene therapy. Also, BRCA1-2 and PTEN were evaluated as potential biomarkers that predict response to PARP inhibitors and mTOR inhibitors respectively. 
With all the above, we limited our little knowledge, to the "mindful" cellular nucleus components, they are primer in Cancer development.

Thereafter many metabolic pathways are adjusted to the new workload of the malignant cell.

Ribosomes increase their number for more protein synthesis (more cytoplasm for the forthcoming cell divisions), some amino acids coming from biochemical altered pathways are clue for they maintain the cancer process.

The endoplasmic reticulum becomes "stressed" and mitochondria's divide(fision ) in order to give more energy (ATP) to the dividing cell.

Considering now all the cell dynamics, the mitotic cell cycle and cell division are the "phenotype" which characterizes Cancer.

Tumors grow by cell divisions, and only some tumors "grow" in their final phases due to the absence of cell loses (accretion).

This process, gives in the space round balls, is not possible to have "other geometries", and present different cell populations and differentiation grades.

The tumors comport different clonal and sub clonal populations that onlywork for the metastatic process. What teleonomic ally speaking means this last process, is a still a great mystery of science.

Malignant tumors only want to continue driving malignancy in other organs far from the primary tumor site, and the only way is to reproduce in every colonized organ, being different balls in size and phases of development (each one earlier than others).

This different "colors" on cells, gives tissue and biochemical heterogeneity to the tumor, that is still the big challenge to resolve in Oncology Precision Medicine. You take with a biopsy from a part aside of other and the first one can be $\mathrm{X}$ and the second one be $\mathrm{Y}$. Also, the oncogenic driven process is perhaps different on each. For the amount of disease in a body, is difficult to truly assess how much of it has X or Y regarding the biopsy And having the mutation may not be a predictive factor for target therapy.

We need the help of Artificial Intelligence to map us every part of the tumor, and after with computational algorithms we can de- cide which drug is best for that patient according mainly the tumor biochemistry. This last is a reality now.

We carry the weight of Cancer since the first multicellular eukaryotic organism. The disease is very complex mainly for it touches the secret of life molecule that is DNA.

In the last 30 years of the last century, the advances were focused mainly in Cancer medical treatment.

And, after this, in the last 20 years of this century, and due to the continuous advances in the basic Cancer Cell knowledge, more precise treatments came into age like the targeted therapy and immunotherapy.

Cancer is not now, the previous picture where the writer writes many letters to the wonderful lady and she doesn't answer.

Now the lady has fallen in love with the novelist, and formally provides him with answers.

The letters text is and will be, our huge basic and therapeutic knowledge in this always challenging disease.

\section{Volume 5 Issue 8 August 2021 (C) All rights are reserved by Daniel Gandia and Juan Manuel Carrera.}

